\title{
Structural analysis for electric circuits and consequences for MNA
}

\author{
D. Estévez Schwarz*and C. Tischendorf ${ }^{\dagger}$
}

\begin{abstract}
The development of integrated circuits requires powerful numerical simulation programs. Of course, there is no method that treats all the different kinds of circuits successfully. The numerical simulation tools provide reliable results only if the circuit model meets the assumptions that guarantee the successful application of the integration software. Because of the large dimension of many circuits (about $10^{7}$ circuit elements) it is often difficult to find the circuit configurations that lead to numerical difficulties. In this paper, we analyze electric circuits with respect to their structural properties in order to give circuit designers some help for fixing modelling problems if the numerical simulation fails. We consider one of the most frequently used modelling technique, the modified nodal analysis (MNA), and discuss the index of the differential algebraic equations (DAEs) obtained by this kind of modelling.
\end{abstract}

Key words: Circuit simulation; differential-algebraic equation; DAE; index; modified nodal analysis; MNA; structural properties; modelling.

AMS Subject Classification: 94C05, 65L05.

\section{Structural analysis}

In the following we discuss lumped electric circuits containing nonlinear and possibly time-variant resistances, capacitances, inductances, voltage sources and current sources. Usually circuit simulation tools are based on these kinds of network elements. For two-terminal (one-port) lumped elements, the current through the element and the voltage across it are well-defined quantities. For lumped elements with more than two terminals, the current entering any terminal and the voltage across any pair of terminals are well defined at all times (cf. [3]). Hence, general n-terminal elements are completely described by $(n-1)$ currents entering the $(n-1)$ terminals and the $(n-1)$ branch voltages across each of these $(n-1)$ terminals and the reference terminal $n$.

\footnotetext{
${ }^{*}$ Humboldt University of Berlin, Germany

$\dagger^{\dagger}$ At present at Lunds University, Sweden
} 


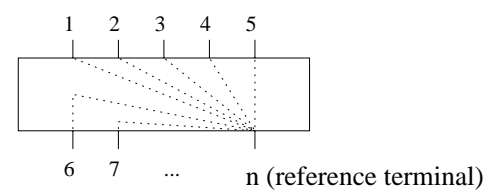

Figure 1.1: n-terminal circuit element

In particular, $n$-terminal resistances can be modeled by an equation system of the form

$$
j_{k}=r_{k}^{e}\left(u_{1}, \ldots, u_{n-1}, t\right) \quad \text { for } \quad k=1, \ldots, n-1
$$

if $j_{k}$ represents the current entering the terminal $k$ and $u_{l}$ describes the voltage across the pair of terminals $\{l, n\}$ (for $k, l=1, \ldots, n-1$ ). The Kirchhoff's Current Law implies the current entering the terminal $n$ to be given by $j_{n}=-\sum_{k=1}^{n-1} j_{k}$. The conductance matrix $G^{e}\left(u_{1}, \ldots, u_{n-1}, t\right)$ is then defined by the Jacobian

$$
G^{e}\left(u_{1}, \ldots, u_{n-1}, t\right):=\left(\begin{array}{ccc}
\frac{\partial r_{1}^{e}}{\partial u_{1}} & \ldots & \frac{\partial r_{1}^{e}}{\partial u_{n-1}} \\
\vdots & \ddots & \vdots \\
\frac{\partial r_{n-1}^{e}}{\partial u_{1}} & \ldots & \frac{\partial r_{n-1}^{e}}{\partial u_{n-1}}
\end{array}\right) .
$$

The index $e$ shall specify the correlation to a special element of a circuit. Later on we will introduce the conductance matrix $G(u, t)$ describing all resistances of a circuit. Correspondingly, the capacitance matrix $C^{e}\left(v_{1}, \ldots, v_{n-1}, t\right)$ of a general $n$-terminal capacitance is given by

$$
C^{e}\left(u_{1}, \ldots, u_{n-1}, t\right):=\left(\begin{array}{ccc}
\frac{\partial q_{1}^{e}}{\partial u_{1}} & \ldots & \frac{\partial q_{1}^{e}}{\partial u_{n-1}} \\
\vdots & \ddots & \vdots \\
\frac{\partial q_{n-1}^{e}}{\partial u_{1}} & \ldots & \frac{\partial q_{n-1}^{e}}{\partial u_{n-1}}
\end{array}\right)
$$

if the voltage-current relation is defined by means of charges by

$$
j_{k}=\frac{d}{d t} q_{k}^{e}\left(u_{1}, \ldots, u_{n-1}, t\right) \quad \text { for } \quad k=1, \ldots, n-1 .
$$

In order to illustrate what the matrices $C^{e}$ may look like, let us consider a MOSFET-model as an example of a common $n$-terminal element.

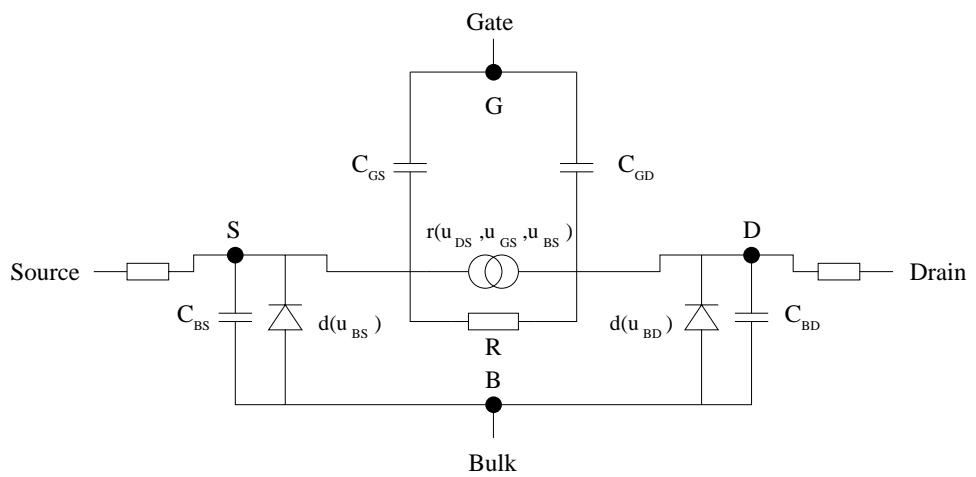

Figure 1.2: MOSFET-model 
Choosing the source node $S$ as the reference node, we have the reference voltages $u_{G S}, u_{D S}$, and $u_{B S}$. For the currents we obtain

$$
\begin{aligned}
j_{G}= & C_{G S} \dot{u}_{G S}+C_{G D}\left(\dot{u}_{G S}-\dot{u}_{D S}\right) \\
j_{D}= & -C_{G D}\left(\dot{u}_{G S}-\dot{u}_{D S}\right)-C_{B D}\left(\dot{u}_{B S}-\dot{u}_{D S}\right) \\
& +d\left(u_{B S}-u_{D S}\right)+g\left(u_{G S}, u_{D S}, u_{B S}\right), \\
j_{B}= & C_{B S} \dot{u}_{B S}+C_{B D}\left(\dot{u}_{B S}-\dot{u}_{D S}\right)-d\left(u_{B S}\right)-d\left(u_{B S}-u_{D S}\right) .
\end{aligned}
$$

Note that $j_{S}$ is given by the formula $j_{S}=-j_{G}-j_{D}-j_{B}$ due to Kirchoff's Current Law. Now it is easy to verify that

$$
C^{e}\left(u_{G S}, u_{D S}, u_{B S}\right)=\left(\begin{array}{ccc}
C_{G S}+C_{G D} & -C_{G D} & 0 \\
-C_{G D} & C_{G D}+C_{B D} & -C_{B D} \\
0 & -C_{B D} & C_{B S}+C_{B D}
\end{array}\right)
$$

for the MOSFET-model from [14].

Inductances can be modeled by means of fluxes by

$$
u_{k}=\frac{d}{d t} \phi_{k}^{e}\left(j_{1}, \ldots, j_{n-1}, t\right) \quad \text { for } \quad k=1, \ldots, n-1 .
$$

Then, the inductance matrix $L^{e}\left(j_{1}, \ldots, j_{n-1}, t\right)$ of a general $n$-terminal inductance is given by the Jacobian

$$
L^{e}\left(j_{1}, \ldots, j_{n-1}, t\right):=\left(\begin{array}{ccc}
\frac{\partial \phi_{1}^{e}}{\partial j_{1}} & \ldots & \frac{\partial \phi_{1}^{e}}{\partial j_{n-1}} \\
\vdots & \ddots & \vdots \\
\frac{\partial \phi_{n-1}^{e}}{\partial j_{1}} & \ldots & \frac{\partial \phi_{n-1}^{e}}{\partial j_{n-1}}
\end{array}\right) .
$$

A commonly used method for network analysis in circuit simulation packages like TITAN ${ }^{1}$ and SPICE is the Modified Nodal Analysis (MNA).

It represents a systematic treatment of general circuits and is important when computers perform the analysis of networks automatically. The scheme to set up the MNA equations is:

1. Write node equations by applying KCL (Kirchhoff's Current Law) to each node except for the datum node:

$$
A j=0 .
$$

The vector $j$ represents the branch current vector. The matrix $A$ is called the (reduced) incidence matrix and describes the network graph, the branch-node relations.

2. Replace the currents $j_{k}$ of voltage-controlled elements by the voltagecurrent relations of these elements in equation (1.1).

3. Add the current-voltage relations for all current-controlled elements.

\footnotetext{
${ }^{1}$ SIEMENS AG.
} 
Note that, in case of multi-terminal elements with $n$ terminals, we speak of branches if they represent a pair of terminals $\{l, n\}$ with $1 \leq l \leq n-1$.

In general, the MNA leads to a coupled system of implicit differential equations and nonlinear equations, i.e., to a differential-algebraic equation (DAE)

$$
f(\dot{x}(t), x(t), t)=0,
$$

where the partial derivative $f_{\dot{x}}^{\prime}(\dot{x}(t), x(t), t)$ is singular. The analytical and numerical solutions of (1.2) depend strongly on its structure and index. For a detailed discussion of this fact we refer to [7], [9], [12], and [13]. Let us note that numerical methods can fail in higher index cases, particularly if the index is greater than 2. Therefore, we are looking for conditions (depending on the network topology) that guarantee a lower index $(\leq 2)$.

In order to obtain more detailed information about the structure of (1.2) we split the (reduced) incidence matrix $A$ into the element-related incidence matrices

$$
A=\left(A_{C}, A_{L}, A_{R}, A_{V}, A_{I}\right),
$$

where $A_{C}, A_{L}, A_{R}, A_{V}$, and $A_{I}$ describe the branch-current relations for capacitive branches, inductive branches, resistive branches, branches of voltage sources and branches of current sources, respectively. Denote by $e$ the node potentials (excepting the datum node) and by $j_{L}$ and $j_{V}$ the current vectors of inductances and voltage sources. Defining the vector of functions for current and voltage sources by $i$ and $v$, respectively, we obtain the following quasi-linear DAE-system from the MNA:

$$
\begin{aligned}
A_{C} \frac{d q\left(A_{C}^{T} e, t\right)}{d t}+A_{R} r\left(A_{R}^{T} e, t\right)+A_{L} j_{L}+A_{V} j_{V} & \\
+A_{I} i\left(A^{T} e, \frac{d q\left(A_{C}^{T} e, t\right)}{d t}, j_{L}, j_{V}, t\right) & =0, \\
\frac{d \phi\left(j_{L}, t\right)}{d t}-A_{L}^{T} e & =0, \\
A_{V}^{T} e-v\left(A^{T} e, \frac{d q\left(A_{C}^{T} e, t\right)}{d t}, j_{L}, j_{V}, t\right) & =0 .
\end{aligned}
$$

Note that the vectors $A_{C}^{T} e, A_{L}^{T} e, A_{R}^{T} e$ and $A_{V}^{T} e$ describe the branch voltages for the capacitive, inductive, resistive and voltage source branches, respectively.

Remark: Due to the fact that the currents through resistances are functions of the branch potentials, we do not include them separately as controlling functions. Of course, if the network does not contain controlled sources, then the source functions reduce to functions $i(t)$ and $v(t)$ which depend on time only.

Nowadays circuit simulation packages use two different approaches for solving (1.3)-(1.5), the conventional and the charge-oriented one.

\section{The conventional MNA}

For the conventional MNA the vector of unknowns consists of all node voltages and all branch currents of current-controlled elements. 
Defining

$C(u, t):=\frac{\partial q(u, t)}{\partial u}, q_{t}^{\prime}(u, t):=\frac{\partial q(u, t)}{\partial t}, L(j, t):=\frac{\partial \phi(j, t)}{\partial j}, \phi_{t}^{\prime}(j, t):=\frac{\partial \phi(j, t)}{\partial t}$

we obtain

$$
\begin{aligned}
A_{C} C\left(A_{C}^{T} e, t\right) A_{C}^{T} \frac{d e}{d t}+A_{C} q_{t}^{\prime}\left(A_{C}^{T} e, t\right)+A_{R} r\left(A_{R}^{T} e, t\right) & \\
+A_{L} j_{L}+A_{V} j_{V}+A_{I} i\left(A^{T} e, C\left(A_{C}^{T} e, t\right) A_{C}^{T} \frac{d e}{d t}, j_{L}, j_{V}, t\right) & =0, \\
L\left(j_{L}, t\right) \frac{d j_{L}}{d t}+\phi_{t}^{\prime}\left(j_{L}, t\right)-A_{L}^{T} e & =0, \\
A_{V}^{T} e-v\left(A^{T} e, C\left(A_{C}^{T} e, t\right) A_{C}^{T} \frac{d e}{d t}, j_{L}, j_{V}, t\right) & =0 .
\end{aligned}
$$

Later on we will also need

$$
G(u, t):=\frac{\partial r(u, t)}{\partial u}, r_{t}^{\prime}(u, t):=\frac{\partial r(u, t)}{\partial t} .
$$

\section{The charge-oriented MNA}

In comparison with the conventional MNA, the vector of unknowns consists additionally of the charge of capacitances and the flux of inductances. Moreover, the original voltage-charge and current-flux equations are added to the system. The resulting system is then of the form (cf. [8])

$$
\begin{aligned}
A_{C} \frac{d q}{d t}+A_{R} r\left(A_{R}^{T} e, t\right)+A_{L} j_{L}+A_{V} j_{V} & \\
+A_{I} i\left(A^{T} e, \frac{d q}{d t}, j_{L}, j_{V}, t\right) & =0, \\
\frac{d \phi}{d t}-A_{L}^{T} e & =0, \\
A_{V}^{T} e-v\left(A^{T} e, \frac{d q}{d t}, j_{L}, j_{V}, t\right) & =0, \\
q-q_{C}\left(A_{C}^{T} e, t\right) & =0, \\
\phi-\phi_{L}\left(j_{L}, t\right) & =0 .
\end{aligned}
$$

\section{Topological characterization of the splitted incidence ma- trix}

The splitting of the incidence matrix $A=\left(A_{C}, A_{L}, A_{R}, A_{V}, A_{I}\right)$ corresponding to certain branches leads to the following useful structural information for lumped circuits:

Theorem 1.1 Given a lumped circuit with capacitances, inductances, resistances, voltage sources and current sources. Then, the following relations are satisfied for the (reduced) incidence matrix $A=\left(A_{C} A_{L} A_{R} A_{V} A_{I}\right)$.

1. Then matrix $\left(A_{C} A_{L} A_{R} A_{V}\right)$ has full row rank, because cutsets of current sources are forbidden. 
2. The matrix $A_{V}$ has full column rank, because loops of voltage sources are forbidden.

3. The matrix $\left(A_{C} A_{R} A_{V}\right)$ has full row rank if and only if the circuit does not contain a cutset consisting of inductances and/or current sources only.

4. Let $Q_{C}$ be any projector onto ker $A_{C}$. Then, the matrix $Q_{C}^{T} A_{V}$ has full column rank if and only if the circuit does not contain a loop consisting of capacitances and voltage sources only.

Note that loops containing only capacitances are excluded under point 4 , whereas cutsets containing only inductances are included under point 3 of Theorem 1.1. For a complete proof of Theorem 1.1 we refer to [16].

In the following the special cutsets and loops considered in Theorem 1.1 will be important. Therefore we define:

1. An L-I cutset is a cutset consisting of inductances and/or current sources only.

2. A C-V loop is a loop consisting of capacitances and voltage sources only.

In order to describe the different parts of the DAE (1.6)-(1.8) in more detail, we will introduce some useful projectors. Before doing this, let us recall the definition of a projector.

Definition 1.2 For $\mathbb{R}^{m}=R_{1} \oplus R_{2}$ a matrix $Q \in \mathbb{R}^{m \times m}$ is a projector onto $R_{1}$ along $R_{2}$ if and only if $Q^{2}=Q, \operatorname{im} Q=R_{1}$, and $\operatorname{ker} Q=R_{2}$.

We denote by $\mathbf{Q}_{\mathbf{C}}, \mathbf{Q}_{\mathbf{V}-\mathbf{C}}, \mathbf{Q}_{\mathbf{R}-\mathbf{C V}}, \mathbf{Q}_{\mathbf{V}}, \overline{\mathbf{Q}}_{\mathbf{C}}$, and $\overline{\mathbf{Q}}_{\mathbf{V}-\mathbf{C}}$ a projector onto $\operatorname{ker} A_{C}^{T}, \operatorname{ker} A_{V}^{T} Q_{C}, \operatorname{ker} A_{R}^{T} Q_{C} Q_{V-C}, \operatorname{ker} A_{V}^{T}, \operatorname{ker} A_{C}$, and $\operatorname{ker} Q_{C}^{T} A_{V}$, respectively. The complementary projectors will be denoted by $\mathbf{P}:=I-Q$, with the corresponding subindex. We observe that

im $P_{C} \subset \operatorname{ker} P_{V-C}, \quad$ im $P_{V-C} \subset \operatorname{ker} P_{R-C V} \quad$ and $\quad$ im $P_{C} \subset \operatorname{ker} P_{R-C V}$,

and that thus $Q_{C} Q_{V-C}$ is a projector onto $\operatorname{ker}\left(A_{C} A_{V}\right)^{T}$, and $Q_{C} Q_{V-C} Q_{R-V C}$ is a projector onto $\operatorname{ker}\left(A_{C} A_{R} A_{V}\right)^{T}$. To shorten denotations, we use the abbreviation $\mathbf{Q}_{\mathbf{C R V}}:=Q_{C} Q_{V-C} Q_{R-C V}$. Remark that the projector $P_{C R V}$ does not coincide with the projector $P_{R-C V}$ in general.

Using the introduced projections we obtain the following corollary from Theorem 1.1.

\section{Corollary 1.3 Theorem 1.1 implies that}

1. $Q_{C R V}=0$ if and only if the network does not contain L-I cutsets,

2. $\bar{Q}_{V-C}=0$ if and only if the network does not contain $C$-V loops.

For a simpler description later on, we adduce two lemmata:

Lemma 1.4 If $M$ is a positive definite $m \times m$-matrix and $N$ is a rectangular matrix of dimension $k \times m$, then it holds that

$$
\operatorname{ker} N M N^{T}=\operatorname{ker} N^{T} \quad \text { and } \quad \operatorname{im} N M N^{T}=\operatorname{im} N .
$$


The correctness of Lemma 1.4 follows immediately from the definition of positive definite matrices.

Lemma 1.5 The matrices

$$
\begin{aligned}
H_{1}\left(A_{C}^{T} e, t\right): & =A_{C} C\left(A_{C}^{T} e, t\right) A_{C}^{T}+Q_{C}^{T} Q_{C}, \\
H_{2}: & =Q_{C}^{T} A_{V} A_{V}^{T} Q_{C}+Q_{V-C}^{T} Q_{V-C}, \\
H_{3}: & =A_{V}^{T} Q_{C} Q_{C}^{T} A_{V}+\bar{Q}_{V-C}^{T} \bar{Q}_{V-C}, \\
H_{4}\left(A_{R}^{T} e\right): & =Q_{V-C}^{T} Q_{C}^{T} A_{R} G\left(A_{R}^{T} e, t\right) A_{R}^{T} Q_{C} Q_{V-C}+Q_{R-C V}^{T} Q_{R-C V}, \\
H_{5}\left(j_{L}, t\right): & =Q_{C R V}^{T} A_{L} L^{-1}\left(j_{L}, t\right) A_{L}^{T} Q_{C R V}+P_{C R V}^{T} P_{C R V}, \\
H_{6}\left(A_{C}^{T} e, t\right): & =\bar{Q}_{V-C}^{T} A_{V}^{T} H_{1}^{-1}\left(A_{C}^{T} e, t\right) A_{V} \bar{Q}_{V-C}+\bar{P}_{V-C}^{T} \bar{P}_{V-C}
\end{aligned}
$$

are regular.

Proof: Using Lemma 1.4, the regularity of $H_{1}\left(A_{C}^{T} e, t\right)$ is obvious since $C\left(A_{C}^{T} e, t\right)$ is positive definite. For $\mathrm{H}_{2}$ and for $\mathrm{H}_{3}$ the regularity follows immediately, and for $H_{4}$ analogously if we consider that $G\left(A_{R}^{T} e, t\right)$ is positive definite.

Let us prove the regularity of $H_{5}$. Let $z$ be an element of ker $H_{5}$. Then we have

$$
\left(Q_{C R V}^{T} A_{L} L^{-1}\left(j_{L}, t\right) A_{L}^{T} Q_{C R V}+P_{C R V}^{T} P_{C R V}\right) z=0 .
$$

If we multiply this equation by $P_{C R V}^{T}$, it results that $P_{C R V}^{T} P_{C R V} z=0$ and, therefore, $P_{C R V} z=0$. Hence, we obtain

$$
Q_{C R V}^{T} A_{L} L^{-1}\left(j_{L}, t\right) A_{L}^{T} Q_{C R V} z=0 .
$$

Then, since $L^{-1}\left(j_{L}, t\right)$ is positive definite, $A_{L}^{T} Q_{C R V} z=0$ holds. Applying that $\left(A_{C}, A_{R}, A_{V}, A_{L}\right)^{T}$ has full column rank, we conclude $Q_{C R V} z=0$, i.e., $z=P_{C R V} z$ and, since $P_{C R V} z=0$, the regularity is verified.

The regularity from $H_{6}\left(A_{C}^{T} e, t\right)$ can be easily shown making use of the facts that $C\left(A_{C}^{T} e, t\right)$ is positive definite and that $A_{V}$ has full column rank.

q.e.d.

\section{The index of DAEs resulting from the MNA for electric circuits}

The numerical behavior of solutions of DAEs depends strongly on their index. Roughly speaking, the index of a DAE is the measure of the deviation of a DAE from regular ODEs. DAEs have, among other things, the following two important properties (see e.g. [1], [6], [10]):

(i) DAEs do not only represent integration problems, but differentiation problems, too. Some parts of a DAE must be differentiable sufficiently often.

(ii) Some components of the solution are determined algebraically. This implies that the choice of initial values is not free for solutions of initial value problems. The initial values must be consistent. 
The higher the index the higher the differentiations needed for solving a DAE. In the nonlinear case, the index concept is not unique in the literature. Therefore we study two important index concepts for the circuits, the differential index in Section 3 and the tractability index in Section 4. The results show that both concepts lead to the same index in case of circuit simulation applying MNA.

The investigations of numerical methods for DAEs have shown that available codes for general nonlinear DAEs provide reliable results only for DAEs of lower index $(\leq 2)$. Therefore we are interested in adequate conditions for electric circuits that guarantee a lower index DAE. In particular, the voltage-controlled voltage sources (VCVS), current-controlled voltage sources (CCVS), voltagecontrolled current sources(VCCS), and current-controlled current sources (CCVS) have to be analyzed very carefully. The result is given in the following theorem.

Theorem 2.1 Consider lumped electric circuits containing resistances, capacitances, inductances, and voltage and current sources. Let the capacitance, inductance and conductance matrices of all capacitances, inductances, and resistances, respectively, be positive definite. ${ }^{2}$ Furthermore, let the following conditions for the controlled sources be satisfied:

1. The controlled voltage sources do not form a part of any $C-V$ loop and their controlling elements fulfill the conditions exposed in the Tables 2.1 and 2.2.

2. Each controlled current source fulfills at least one of the following conditions:

(a) It does not form a part of any L-I cutset and the controlling elements fulfill the conditions exposed in the Tables 2.3 and 2.4.

(b) There exists a path formed by capacitances that connects its incidence nodes. The controlling elements fulfill the conditions exposed in Table 2.5 for CCCS, and the VCCS are controlled by an arbitrary voltage.

(c) There exists a path formed by capacitances and voltage sources that connects its incidence nodes. The controlling elements fulfill the conditions exposed in Table 2.6 for CCCS, and the VCCS are controlled by an arbitrary voltage.

Then, the conventional MNA leads to an index-1 DAE $E^{3}$ if and only if the network contains neither $L-I$ cutsets nor $C$ - $V$ loops. Otherwise, the conventional MNA leads to an index-2 DAE.

Theorem 2.2 Theorem 2.1 holds if we consider the charge-oriented MNA instead of the conventional MNA.

\section{Remarks:}

1. Similar results are well-known for the state equations of dynamic linear networks (see e.g. [2]).

\footnotetext{
${ }^{2}$ For capacitances and inductances with affine characteristics the positive definiteness implies that they are strictly locally passive (cf. [5]).

${ }^{3}$ For reasons of simplicity, we do not consider the index-0 cases, which result if $f_{\dot{x}}^{\prime}(\dot{x}(t), x(t), t)$ is regular, separately.
} 
2. For linear networks with special controlled sources, this coincides with results in [13].

3. The presented criteria can be checked locally. It is neither necessary to find convenient trees nor to make additional assumptions on the functions and parameters that define the controlled sources. Usually, it is not difficult to check whether a model of a network element including controlled sources satisfies these conditions or not.

4. If a model of a network element does not satisfy the conditions, it is not difficult to fulfill them by introducing some additional capacitances, resistances or inductances.

5. Nevertheless, the topological assumptions made for the controlled sources are sufficient but not necessary.

\section{Examples:}

1. Consider again the MOSFET-model given in Figure 1.2. The VCCS from source to drain is controlled by the branch voltages $u_{G S}, u_{D S}$, and $u_{B S}$. For this, the conditions (2a)-(2c) are satisfied since there are capacitive ways from gate to source, from drain to source as well as from bulk to source, and there exists a capacitive way from source to drain.

2. Consider the VCCS in Figure 2.1 (from [2]). The considered CCVS does not form a part of a C-V-loop and it is controlled by the current of a branch that forms a cutset with inductances. Therefore, it meets the condition (1) of Theorem 2.1.

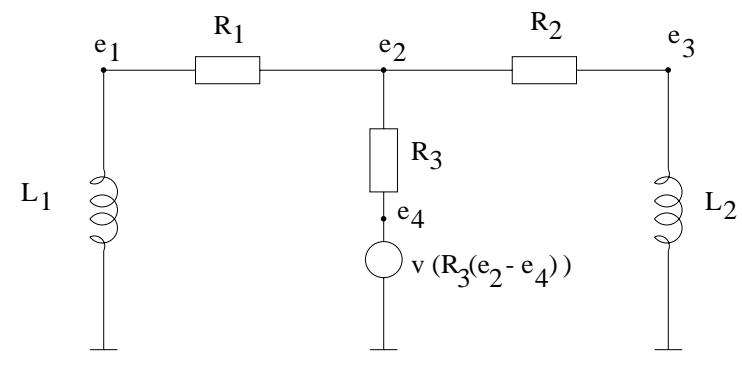

Figure 2.1: Circuit with CCVS

Corollary 2.3 The assumption of Theorem 2.1 on the resistances can be slightly reduced. In fact, only the positive definiteness of the conductance matrix corresponding to those resistances that do not form a loop with capacitances and/or voltage sources is required.

This statement follows immediately from Theorem 2.1 if we consider the resistances as VCCS.

In order to obtain a description of assumption (1) by means of projectors, we split the incidence matrix $A_{V}$ into $\left(A_{V t} A_{V c o}\right)$ for independent and controlled sources, respectively. 
The controlling voltages of a VCVS can be voltages of:

1. capacitances,

2. independent voltage sources,

3. CCVSs that are controlled by:

(a) inductances,

(b) independent current sources,

(c) resistances or VCCSs for which the controlling nodes are connected by:

i. capacitances,

ii. independent voltage sources,

iii. paths containing only the elements described in $(3(\mathrm{c}) \mathrm{i})$, (3(c)ii),

(d) branches that form a cutset with the elements described in (3a), $(3 \mathrm{~b})$ and $(3 \mathrm{c})$,

4. branches that form a loop with the elements described in (1), (2) and (3).

Table 2.1: VCVS - condition (1)

Lemma 2.4 The condition that controlled voltage sources do not form a part of a $C$-V loop is equivalent to $\bar{Q}_{V-C}=\left(\begin{array}{c}\left(\bar{Q}_{V-C}\right)_{t} \\ 0\end{array}\right)$. Here, $\left(\bar{Q}_{V-C}\right)_{t}$ denotes the upper part of $\bar{Q}_{V-C}$ corresponding to $A_{V t}$.

Proof: A controlled voltage source forms a part of a C-V loop if and only if the column $a_{s}$ of $A_{V c o}$ corresponding to this source depends linearly on the columns of $\left(A_{C} \hat{A}_{V}\right)$, where $\hat{A}_{V}$ denotes the matrix $A_{V}$ reduced by the column $a_{s}$, i.e., there is a vector $v$ such that

$$
\left(A_{C} A_{V}\right) v=0 \quad \text { and } \quad v_{s} \neq 0
$$

for the $s$-th component of $v$ corresponding to the controlled source considered. That means, there is a vector $v$ such that

$$
Q_{C}^{T} A_{V} v=0 \text { and } v_{s} \neq 0,
$$

i.e., the $s$-th row of $\bar{Q}_{V-C}$ has a non-zero entry. This is equivalent to

$$
\bar{Q}_{V-C} \neq\left(\begin{array}{c}
\left(\bar{Q}_{V-C}\right)_{t} \\
0
\end{array}\right) \text {. }
$$

q.e.d.

Hence, assumption (1) of Theorem 2.1 implies that

$$
\begin{aligned}
\bar{Q}_{V-C}^{T} v\left(A^{T} e, \frac{d q\left(A_{C}^{T} e, t\right)}{d t}, j_{L}, j_{V}, t\right) & =\bar{Q}_{V-C}^{T} v_{t}(t), \\
v\left(A^{T} e, \frac{d q\left(A_{C}^{T} e, t\right)}{d t}, j_{L}, j_{V}, t\right) & =v_{*}\left(A_{C}^{T} e, j_{L}, t\right)
\end{aligned}
$$


The controlling currents of a CCVS can be currents of:

1. inductances,

2. independent current sources,

3. resistances or VCCSs for which the controlling nodes are connected by:

(a) capacitances,

(b) independent voltage sources,

(c) VCVSs for which the nodes that incide with the controlling branch are connected by

i. capacitances,

ii. independent voltage sources,

iii. paths containing only the elements described in (3(c)i), (3(c)ii),

(d) paths containing only the elements described in (3a), (3b) and (3c),

4. branches that form a cutset with the elements described in (1), (2) and (3).

Table 2.2: CCVS - condition (1)

The controlling voltages of a VCCS can be voltages of:

1. capacitances,

2. voltage sources,

3. branches that form a loop with branches like those described in (1) and (2).

Table 2.3: VCCS - condition (2a)

for a suitable function $v_{*}$ and for a vector $v_{t}(t)$ that contains the functions of independent voltage sources and zeros instead of the functions of controlled voltage sources. In the following we will drop the index *.

In order to transcribe the assumptions made for controlled current sources, we split the incidence matrix $A_{I}$ into $\left(A_{I t}, A_{I a}, A_{I b}, A_{I c}\right)$ and the current vector $i$ correspondingly, for the independent current sources and the controlled current sources that fulfill $(2 \mathrm{a}),(2 \mathrm{~b})$ and $(2 \mathrm{c})$, respectively. If a controlled current source fulfills more than one of the conditions $(2 \mathrm{a}),(2 \mathrm{~b})$ and $(2 \mathrm{c})$, the corresponding column of $A_{I}$ should be assigned to only one of the matrices $A_{I a}, A_{I b}$, and $A_{I c}$.

Lemma 2.5 The condition that controlled current sources do not form a part of an L-I cutset is equivalent to the relation $Q_{C R V}^{T} A_{I}=\left(Q_{C R V}^{T} A_{I t} 0\right)$.

Proof: A controlled current source forms a part of an L-I cutset if and only if the column $a_{s}$ of $\left(A_{I a}, A_{I b}, A_{I c}\right)$ corresponding to this controlled source is 
The controlling currents of a CCCS can be currents of:

1. inductances,

2. independent current sources,

3. resistances or VCCSs for which the controlling nodes are connected by:
(a) capacitances,
(b) voltage sources,
(c) paths containing only the elements described in (3a) and (3b),

4. branches that form a cutset with the elements described in (1), (2) or (3).

Table 2.4: CCCS - condition (2a)

The controlling current of a CCCS can be the current of:

1. inductances,

2. independent current sources,

3. resistances,

4. voltage sources that do not form a part of a $\mathrm{C}-\mathrm{V}$ loop,

5. VCCS,

6. a branch that forms a cutset with the elements described in (1), (2), (3), (4) and (5).

Table 2.5: CCCS - condition (2b)

linearly independent of the columns belonging to $\left(A_{C}, A_{R}, A_{V}\right)$, i.e.,

$$
a_{s} \notin \operatorname{im}\left(A_{C} A_{R} A_{V}\right) \text { and, therefore, } Q_{C R V}^{T} a_{s} \neq 0 .
$$

But, this is equivalent to the condition that $Q_{C R V}^{T}\left(A_{I a}, A_{I b}, A_{I c}\right) \neq 0$.

$$
\text { q.e.d. }
$$

Thus, assumption (2a) of Theorem 2.1 implies that

$$
\begin{aligned}
Q_{C R V}^{T} A_{I} i\left(A^{T} e, \frac{d q\left(A_{C}^{T} e, t\right)}{d t}, j_{L}, j_{V}, t\right) & =Q_{C R V}^{T} A_{I t} i_{t} \\
i\left(A^{T} e, \frac{d q\left(A_{C}^{T} e, t\right)}{d t}, j_{L}, j_{V}, t\right) & =i_{a}\left(A_{C}^{T} e, A_{V}^{T} e, j_{L}, t\right)
\end{aligned}
$$

for a suitable function $i_{a}$.

Furthermore, assumption (2b) of Theorem 2.1 implies by definition that

$$
\begin{aligned}
Q_{C}^{T} A_{I b} & =0, \\
i\left(A^{T} e, \frac{d q\left(A_{C}^{T} e, t\right)}{d t}, j_{L}, j_{V}, t\right) & =i_{b}\left(A^{T} e, j_{L}, \bar{P}_{V-C} j_{V}, t\right)
\end{aligned}
$$


The controlling current of a CCCS can be the current of:

1. inductances,

2. resistances,

3. independent current sources,

4. VCCS,

5. a branch that forms a cutset with the elements described in (1), (2), (3) and (4).

Table 2.6: CCCS - condition (2c)

for a suitable function $i_{b}$.

Finally, assumption (2c) of Theorem 2.1 implies that

$$
\begin{aligned}
Q_{V-C}^{T} Q_{C}^{T} A_{I c} & =0, \\
i\left(A^{T} e, \frac{d q\left(A_{C}^{T} e, t\right)}{d t}, j_{L}, j_{V}, t\right) & =i_{c}\left(A^{T} e, j_{L}, t\right)
\end{aligned}
$$

for a suitable function $i_{c}$.

Regarding (2.3), (2.5), and (2.7), the assumptions imply that

$$
Q_{C R V}^{T} A_{I} i\left(A^{T} e, \frac{d q\left(A_{C}^{T} e, t\right)}{d t}, j_{L}, j_{V}, t\right)=Q_{C R V}^{T} A_{I t} i_{t}
$$

is always fulfilled. To shorten denotations we write

$$
i\left(A^{T} e, j_{L}, P_{V-C} j_{V}, t\right)
$$

when we do not distinguish between (2.4), (2.6), and (2.8).

The proofs of the theorems follow in the next sections.

\section{The differential index}

\subsection{Definition of the differential index}

The most general definition of the differential index of nonlinear DAE systems is (cf. [1] pp. 32-33):

Definition 3.1 The differential index $\nu$ of the general nonlinear, sufficiently smooth DAE

$$
f\left(x^{\prime}, x, t\right)=0
$$


is the smallest $\nu$ such that

$$
\begin{aligned}
f\left(x^{\prime}, x, t\right) & =0, \\
\frac{d}{d t} f\left(x^{\prime}, x, t\right) & =0, \\
& \cdot \\
& \cdot \\
\frac{d^{\nu}}{d t^{\nu}} f\left(x^{\prime}, x, t\right) & =0
\end{aligned}
$$

uniquely determines the variable $x^{\prime}$ as a continuous function of $(x, t)$.

Fortunately, the structure of the DAEs that results from the MNA in circuit simulation is such that it will not be necessary to derive the whole function $f$. As we will see, it suffices to derive the explicit constraints in the index 1 case and, additionally, the hidden constraints in the index 2 case.

\subsection{The differential index of the DAE systems in circuit simulation}

In this section we obtain the differential index of the DAE system as well as expressions for the constraints. In the following we assume that the required smoothness is given.

Theorem 3.2 Consider lumped electric circuits satisfying the assumptions of Theorem 2.1. Then it holds:

1. If the network contains neither L-I cutsets nor C-V loops, then the conventional MNA leads to a DAE system with differential index-1 and the constraints are only the explicit ones:

$$
\begin{aligned}
Q_{C}^{T}\left[A_{R} r\left(A_{R}^{T} e, t\right)+A_{L} j_{L}+A_{V} j_{V}+A_{I a, c} i_{a, c}\left(A^{T} e, j_{L}, t\right)\right] & =0 \\
A_{V}^{T} e-v\left(A_{C}^{T} e, j_{L}, t\right) & =0 .
\end{aligned}
$$

2. If the network contains $L-I$ cutsets or $C$-V loops, then the conventional $M N A$ leads to a DAE system with differential index-2. With regard to the constraints, we distinguish the following three possibilities.

(a) If the network does not contain an L-I cutset (but contains $C$-V loops), then the constraints are the explicit ones, namely (3.2) and (3.3), and, additionally, the hidden constraint:

$$
\begin{gathered}
\bar{Q}_{V-C}^{T} A_{V}^{T} H_{1}^{-1}\left(A_{C}^{T} e, t\right) P_{C}^{T}\left[A_{C} q_{t}^{\prime}\left(A_{C}^{T} e, t\right)+A_{R} r\left(A_{R}^{T} e, t\right)+A_{L} j_{L}\right. \\
\left.+A_{V} j_{V}+A_{I} i\left(A^{T} e, j_{L}, \bar{P}_{V-C} j_{V}, t\right)\right]+\bar{Q}_{V-C}^{T} \frac{d v_{t}}{d t}=0 .
\end{gathered}
$$

(b) If the network does not contain $C$-V loops, but contains L-I cutsets, the constraints are the explicit ones, (3.2) and (3.3), and, additionally, the hidden constraint:

$$
Q_{C R V}^{T}\left(A_{L} L^{-1}\left(j_{L}, t\right)\left(A_{L}^{T} e-\phi_{t}^{\prime}\left(j_{L}, t\right)\right)+A_{I t} \frac{d i_{t}}{d t}\right)=0 .
$$


(c) If the network contains $L-I$ cutsets and $C$ - $V$ loops, then the $M N A$ leads to a DAE system with differential index-2. In this case, the constraints are the explicit ones, (3.2) and (3.3), and the hidden ones (3.4) and (3.5). ${ }^{4}$

Remember that the functions $v_{t}(t)$ and $i_{t}(t)$ represent the function of the independent voltage sources and that the matrices $H_{1}(\cdot)-H_{6}(\cdot)$ were defined in Lemma 1.5.

Proof: In the following we will take advantage of the fact that the analyzed system is quasi-linear and that the matrices $C\left(A_{C}^{T} e, t\right), L\left(j_{L}, t\right)$ and $G\left(A_{R}^{T} e, t\right)$ are positive definite. Our aim is to obtain a representation of $\frac{d e}{d t}, \frac{d j_{L}}{d t}$, $\frac{d j_{V}}{d t}$ as continuous functions of $e, j_{L}$ and $j_{V}$. To this purpose, we consider the following splittings:

$$
\begin{aligned}
\frac{d e}{d t} & =P_{C} \frac{d e}{d t}+Q_{C} P_{V-C} \frac{d e}{d t}+Q_{C} Q_{V-C} P_{R-C V} \frac{d e}{d t}+Q_{C R V} \frac{d e}{d t} \\
\frac{d j_{V}}{d t} & =\bar{P}_{V-C} \frac{d j_{V}}{d t}+\bar{Q}_{V-C} \frac{d j_{V}}{d t} .
\end{aligned}
$$

First we make a general approach and afterwards we distinguish the different cases with regard to the topological properties of the network.

$\underline{\underline{\text { Step } 0}}$

If we multiply equation (1.6) by $H_{1}^{-1}\left(A_{C}^{T}, t\right) P_{C}^{T}$ and $Q_{C}^{T}$, respectively, we obtain

$$
\begin{aligned}
P_{C} \frac{d e}{d t}= & -H_{1}^{-1}\left(A_{C}^{T} e, t\right) P_{C}^{T}\left[A_{C} q_{t}^{\prime}\left(A_{C}^{T} e, t\right)+A_{R} r\left(A_{R}^{T} e, t\right)+A_{L} j_{L}\right. \\
& \left.+A_{V} j_{V}+A_{I} i\left(A^{T} e, j_{L}, P_{V-C} j_{V}, t\right)\right]
\end{aligned}
$$

and (3.2). As $L\left(j_{L}, t\right)$ is regular, we obtain equations for $\frac{d j_{L}}{d t}$ directly from (1.7)

$$
\frac{d j_{L}}{d t}=L^{-1}\left(j_{L}, t\right)\left(A_{L}^{T} e-\phi_{t}^{\prime}\left(j_{L}, t\right)\right)
$$

Note that the arguments of the controlled sources in (3.6), (3.2), and (3.3) are written in accordance with (2.2) - (2.10).

$\underline{\underline{\text { Step } 1}}$

Next we differentiate the equations (3.3) and (3.2), i.e., the constraints, and split them in the following way:

Step 1a

Using (2.1) we split the derivative of (3.3) into

$$
\begin{aligned}
\bar{Q}_{V-C}^{T} A_{V}^{T} P_{C} \frac{d e}{d t} & =\bar{Q}_{V-C}^{T} \frac{d v_{t}}{d t} \text { and } \\
\bar{P}_{V-C}^{T} A_{V}^{T}\left(P_{C}+Q_{C}\right) \frac{d e}{d t} & =\bar{P}_{V-C}^{T} \frac{d v\left(A_{C}^{T} e, j_{L}, t\right)}{d t} .
\end{aligned}
$$

\footnotetext{
${ }^{4}$ The expressions for the explicit and the hidden constraints are of special interest with regard to a consistent initialization (cf. [4]).
} 
If we consider (3.8), we can realize that $P_{C} \frac{d e}{d t}$ can be substituted making use of (3.6) to achieve the hidden constraint (3.4).

Step $1 b$

Using (2.9) we split the derivative of (3.2) into

$$
\begin{gathered}
Q_{C R V}^{T}\left[A_{L} \frac{d j_{L}}{d t}+A_{I t} \frac{d i_{t}}{d t}\right]=0 \\
P_{R-C V}^{T} Q_{V-C}^{T} Q_{C}^{T}\left[A_{R} G\left(A_{R}^{T} e, t\right) A_{R}^{T} \frac{d e}{d t}+A_{R} r_{t}^{\prime}\left(A_{R}^{T} e, t\right)\right. \\
\left.+A_{L} \frac{d j_{L}}{d t}+A_{I a} \frac{d i_{a}\left(A_{C}^{T} e, A_{V}^{T} e, j_{L}, t\right)}{d t}\right]=0
\end{gathered}
$$

and

$$
\begin{aligned}
& P_{V-C}^{T} Q_{C}^{T}\left[A_{R} G\left(A_{R}^{T} e, t\right) A_{R}^{T} \frac{d e}{d t}+A_{R} r_{t}^{\prime}\left(A_{R}^{T} e, t\right)+A_{L} \frac{d j_{L}}{d t}\right. \\
&\left.+A_{V} \frac{d j_{V}}{d t}+A_{I a, c} \frac{d i_{a, c}\left(A^{T} e, j_{L}, t\right)}{d t}\right]=0
\end{aligned}
$$

Taking into account (3.7), (3.10) leads to the hidden constraint (3.5).

Step 2

Finally we differentiate the two hidden constraints (3.5) and (3.4) obtained in the last step:

$$
\begin{aligned}
& \frac{d}{d t}\left(Q_{C R V}^{T} A_{L} L^{-1}\left(j_{L}, t\right)\left(A_{L}^{T} e-\phi_{t}^{\prime}\left(j_{L}, t\right)\right)\right) \\
&+Q_{C R V}^{T} A_{I t} \frac{d^{2} i_{t}}{d t^{2}}=0
\end{aligned}
$$

and

$$
\begin{aligned}
& \frac{d}{d t}\left(\overline { Q } _ { V - C } ^ { T } A _ { V } ^ { T } H _ { 1 } ^ { - 1 } ( A _ { C } ^ { T } e , t ) P _ { C } ^ { T } \left[A_{C} q_{t}^{\prime}\left(A_{C}^{T} e, t\right)+A_{R} r\left(A_{R}^{T} e, t\right)\right.\right. \\
& \left.\left.+A_{L} j_{L}+A_{V} j_{V}+A_{I} i\left(A^{T} e, j_{L}, \bar{P}_{V-C} j_{V}, t\right)\right]\right)+\bar{Q}_{V-C}^{T} \frac{d^{2} v_{t}}{d t^{2}}=0
\end{aligned}
$$

Step 3

Let us now take into account the different topological properties of the systems we mentioned in Theorem 2.1:

1. If the network does not contain L-I cutsets, then $Q_{C R V}=0$ (cf. point 3 in Theorem 1.1). Thus, in this case there is no hidden constraint (3.5). Moreover, as we have $\frac{d e}{d t}=P_{C} \frac{d e}{d t}+Q_{C} P_{V-C} \frac{d e}{d t}+Q_{C} Q_{V-C} P_{R-C V} \frac{d e}{d t}$ then, already Step 1 leads to an expression for $\frac{d e}{d t}$. If we multiply (3.9) by $H_{2}^{-1} Q_{C}^{T} A_{V}$ after substituting the expressions (3.6) for $P_{C} \frac{d e}{d t}$ and (3.7) for $\frac{d j_{L}}{d t}$, we obtain an expression for $P_{V-C} \frac{d e}{d t}$. Then, substituting $P_{C} \frac{d e}{d t}+Q_{C} P_{V-C} \frac{d e}{d t}$ and $\frac{d j_{L}}{d t}$ into (3.11) and multiplying by $H_{4}^{-1}(\cdot)$, we obtain a representation for $P_{R-C V} \frac{d e}{d t}$.

Note that these transformations are reversible by multiplication by $H_{3}^{-1} A_{V}^{T} Q_{C} H_{2}$ and $H_{4}(\cdot)$, respectively. 
2. If the network contains an L-I cutset, then $Q_{C R V} \neq 0$ (cf. point 3 in Theorem 1.1). Therefore, we consider

$$
\frac{d e}{d t}=P_{C} \frac{d e}{d t}+Q_{C} P_{V-C} \frac{d e}{d t}+Q_{C} Q_{V-C} P_{R-C V} \frac{d e}{d t}+Q_{C R V} \frac{d e}{d t}
$$

and observe that we obtain the needed expression for $Q_{C R V} \frac{d e}{d t}$ when multiplying (3.13) by $H_{5}^{-1}(\cdot)$ after substituting the expressions for $P_{C} \frac{d e}{d t}+$ $Q_{C} P_{V-C} \frac{d e}{d t}+Q_{C} Q_{V-C} P_{R-C V} \frac{d e}{d t}$ and $\frac{d j_{L}}{d t}$.

3. If the network does not contain C-V loops, then $Q_{C}^{T} A_{V}$ has full column rank (cf. point 4 in Theorem 1.1). Therefore, $\bar{P}_{V-C}=I$, and we obtain an expression for $\frac{d j_{V}}{d t}$ when multiplying (3.12) by $H_{3}^{-1} A_{V}^{T} Q_{C}$ after substituting the obtained expressions for $\frac{d e}{d t}$ and $\frac{d j_{L}}{d t}$. This transformation is reversible as well, as can be seen by multiplication by $H_{2}^{-1} Q_{C}^{T} A_{V} H_{3}$. On the other hand, as $\bar{Q}_{V-C}=0$, there is no hidden constraint (3.4).

4. If the network contains a C-V loop, then $Q_{C}^{T} A_{V}$ does not have full column rank (cf. point 4 in Theorem 1.1). Therefore, $\bar{Q}_{V-C} \neq 0$, and we obtain an expression for $\bar{Q}_{V-C} \frac{d j_{V}}{d t}$ from (3.14) by multiplication by $H_{6}^{-1}(\cdot)$ after the substitution of $\frac{d e}{d t}$, $\frac{d j_{L}}{d t}$ and $\bar{P}_{V-C} \frac{d j_{V}}{d t}$.

Note that this is successively possible because of (2.3)-(2.8) and that it is important to achieve first the complete expression for $\frac{d e}{d t}$ and afterwards those for $\frac{d j_{V}}{d t}$, because of the allowed controlling elements in $(2 \mathrm{c}) .^{5}$

Step 4

Now we analyze the possible cases:

1. If the network contains neither L-I cutsets nor controlled C-V loops, both equations, (3.4) and (3.5), do not appear, i.e., we obtain a representation for $\frac{d e}{d t}, \frac{d j_{L}}{d t}, \frac{d j_{V}}{d t}$ as functions of $e, j_{L}$ and $j_{V}$ with the expressions obtained in Step 1. Thus, the differential index of the system is 1 and no hidden constraints appear.

2. In the other three cases, one more differentiation has to be carried out in order to find explicit expressions for the derivatives. Therefore, the differential index is 2 .

With Step 3 the statements of the theorem follow immediately from Step 2.

$$
\text { q.e.d. }
$$

Theorem 3.3 If the differential index is 1, then the network contains neither $C$ - $V$ loops nor $L-I$ cutsets. If the differential index is 2, then the network contains at least a $C$ - $V$ loop or an L-I cutset.

Proof: Let us now suppose that the differential index is 1 . Then the hidden constraints have to be trivial, i.e., if we regard the homogeneous system, we

\footnotetext{
${ }^{5}$ This variation of the order changes the spaces associated with the DAE-system, as will be shown in Lemma 4.4 .
} 
obtain

$$
\begin{aligned}
\bar{Q}_{V-C}^{T} A_{V}^{T} P_{C} \frac{d e}{d t} & =0 \\
Q_{C R V}^{T} A_{L} \frac{d j_{L}}{d t} & =0 .
\end{aligned}
$$

Making use of the fact that $A_{V}^{T}$ and $\left(A_{C}, A_{R}, A_{V}, A_{L}\right)$ have full row rank, we obtain $\bar{Q}_{V-C}=0$ and $Q_{C R V}=0$ (cf. 4 and 3 in Theorem 1.1), i.e., the network does not contain $\mathrm{C}-\mathrm{V}$ loops or L-I cutsets.

If the index is supposed to be 2, then at least one constraint has to appear, i.e., either $\bar{Q}_{V-C}$ or $Q_{C R V}$ has to be nontrivial (or both). Again, Theorem 1.1 implies that the network has to contain at least a C-V loop or an L-I cutset.

q.e.d.

\section{Proof of Theorem 2.2:}

To conclude, we observe that Theorem 3.2 holds analogously for the chargeoriented MNA. The results obtained with the differential index method are basically the same as those for the conventional MNA. To shorten denotations, we drop the arguments of the controlled sources, because they appear in the same way as in the proof of Theorem 3.2. Analogously to Step 0 from the proof of Theorem 2.1, we can split (1.9) into

$$
\begin{aligned}
A_{C} \frac{d q}{d t}+P_{C}^{T}\left(A_{R} r\left(A_{R}^{T} e, t\right)+A_{L} j_{L}+A_{V} j_{V}+A_{I} i\right) & =0 \\
Q_{C}^{T}\left(A_{R} r\left(A_{R}^{T} e, t\right)+A_{L} j_{L}+A_{V} j_{V}+A_{I} i\right) & =0 .
\end{aligned}
$$

If we define $\bar{Q}_{C}$ as a projector onto $\operatorname{ker} A_{C}$, we can define the matrix $\bar{H}_{C 1}$ := $A_{C}^{T} A_{C}+\bar{Q}_{C}^{T} \bar{Q}_{C}$, which is regular, and obtain the expression

$$
\bar{P}_{C} \frac{d q}{d t}=-\bar{H}_{C 1}^{-1} A_{C}^{T} P_{C}^{T}\left(A_{R} r\left(A_{R}^{T} e, t\right)+A_{L} j_{L}+A_{V} j_{V}+A_{I} i\right)
$$

by multiplication of (3.17) by $\bar{H}_{C 1}^{-1} A_{C}^{T}$. Note that this transformation is reversible by multiplication by $\bar{H}_{C 2}^{-1} A_{C} A_{C}^{T} A_{C}$, if $\bar{H}_{C 2}:=A_{C} A_{C}^{T}+Q_{C}^{T} Q_{C}$. As equation (1.10) is already an expression for $\frac{d \phi}{d t}$, the constraints are (1.11)-(1.13) and (3.18). The derivatives of (1.12) and (1.13) can be splitted as follows:

$$
\begin{aligned}
\bar{P}_{C} \frac{d q}{d t}-\bar{P}_{C} C\left(A_{C}^{T} e, t\right) A_{C}^{T} \frac{d e}{d t}-\bar{P}_{C} q_{t}^{\prime}\left(A_{C}^{T} e, t\right) & =0, \\
\bar{Q}_{C} \frac{d q}{d t}-\bar{Q}_{C} C\left(A_{C}^{T} e, t\right) A_{C}^{T} \frac{d e}{d t}-\bar{Q}_{C} q_{t}^{\prime}\left(A_{C}^{T} e, t\right) & =0, \\
\frac{d \phi}{d t}-L\left(j_{L}, t\right) \frac{d j_{L}}{d t}-\phi_{t}^{\prime}\left(j_{L}, t\right) & =0 .
\end{aligned}
$$

From (3.19) we obtain the following expression for $P_{C} \frac{d e}{d t}$ and multiplication by $H_{1}^{-1}\left(A_{C}^{T} e, t\right) A_{C}$

$$
\begin{aligned}
P_{C} \frac{d e}{d t}= & -H_{1}^{-1}\left(A_{C}^{T} e, t\right) P_{C}^{T}\left(A_{R} r\left(A_{R}^{T} e, t\right)+A_{L} j_{L}+A_{V} j_{V}+A_{I} i\right) \\
& -H_{1}^{-1}\left(A_{C}^{T} e, t\right) A_{C} q_{t}^{\prime}\left(A_{C}^{T} e, t\right) .
\end{aligned}
$$


Note that this holds because of

$$
H_{1}^{-1}(\cdot) A_{C} \bar{H}_{C 1}^{-1} A_{C}^{T} P_{C}^{T}=H_{1}^{-1}(\cdot) \overbrace{A_{C} \underbrace{\bar{H}_{C 1}^{-1} A_{C}^{T} A_{C}}_{\bar{P}_{C}} A_{C}^{T} \bar{H}_{C 2}^{-1}}^{P_{C}^{T}} P_{C}^{T}=H_{1}^{-1}(\cdot) P_{C}^{T} .
$$

Inserting this into (3.20), we obtain an equation for $\bar{Q}_{C} \frac{d q}{d t}$. On the other hand, (3.21) leads to the expression (3.7) for $\frac{d j_{L}}{d t}$. As the constraints (1.11) and (3.18) are the same as (3.2) and (3.3) in the conventional MNA, the expressions for the remaining derivatives are identical. This implies that the index statements of Theorem (3.2) are valid for the charge-oriented MNA, too.

$$
\text { q.e.d. }
$$

Remark: Observe that only the required smoothness has to be given in each case, and that we can recognize the smoothness requirements directly in the above equations. The next chapter shows how it is possible to define an index with considerably fewer smoothness assumptions on the variables and on the input functions. This is specially relevant for circuit simulation because, in general, only low smoothness is given.

\section{The tractability index}

\subsection{Definition of the tractability index}

The tractability index ([6], [11]) orientates on the linearization of a DAE. This index concept requires only weak smoothness conditions. Furthermore, solvability and stability results exist for index-1-tractable and index-2-tractable DAEs (see e.g. [12], [15]).

We consider nonlinear DAEs

$$
f\left(x^{\prime}, x, t\right)=0
$$

for which $N:=\operatorname{ker} f_{x^{\prime}}^{\prime}\left(x^{\prime}, x, t\right)$ is constant and $f$ is continuously differentiable. We denote $A\left(x^{\prime}, x, t\right):=f_{x^{\prime}}^{\prime}\left(x^{\prime}, x, t\right)$ and $B\left(x^{\prime}, x, t\right):=f_{x}^{\prime}\left(x^{\prime}, x, t\right)$.

Definition 4.1 The DAE (4.1) is called index-1-tractable if the matrix $A_{1}\left(x^{\prime}, x, t\right):=A\left(x^{\prime}, x, t\right)+B\left(x^{\prime}, x, t\right) Q$ is regular for a constant projector $Q$ onto $N$.

\section{Remarks:}

1. The matrix $A_{1}\left(x^{\prime}, x, t\right)$ is regular if and only if $N \cap S\left(x^{\prime}, x, t\right)=\{0\}$ for $S\left(x^{\prime}, x, t\right):=\left\{z: B\left(x^{\prime}, x, t\right) z \in \operatorname{im} A\left(x^{\prime}, x, t\right)\right\}$.

2. The condition does not depend on the choice of the projector $Q$.

For a proof see e.g. [6].

Definition 4.2 The DAE (4.1) is called index-2-tractable if

1. it is not index-1-tractable, 
2. $N_{1}\left(x^{\prime}, x, t\right):=\operatorname{ker} A_{1}\left(x^{\prime}, x, t\right)$ is of constant rank,

3. $A_{2}\left(x^{\prime}, x, t\right):=A_{1}\left(x^{\prime}, x, t\right)+B_{1}\left(x^{\prime}, x, t\right) Q_{1}\left(x^{\prime}, x, t\right)$ is regular for a projector $Q_{1}\left(x^{\prime}, x, t\right)$ onto $N_{1}\left(x^{\prime}, x, t\right)$ and $B_{1}\left(x^{\prime}, x, t\right):=B\left(x^{\prime}, x, t\right)(I-Q)$.

\section{Remarks:}

1. The matrix $A_{2}\left(x^{\prime}, x, t\right)$ is regular iff $N_{1}\left(x^{\prime}, x, t\right) \cap S_{1}\left(x^{\prime}, x, t\right)=\{0\}$ for $S_{1}\left(x^{\prime}, x, t\right):=\left\{z: B_{1}\left(x^{\prime}, x, t\right) z \in \operatorname{im} A_{1}\left(x^{\prime}, x, t\right)\right\}$.

2. The condition does not depend on the choice of the projector $Q_{1}$.

For a proof see again [6].

\subsection{The tractability index of the DAE systems in circuit simulation}

Note that the assumption $N:=\operatorname{ker} f_{x^{\prime}}^{\prime}\left(x^{\prime}, x, t\right)$ is constant is given for the quasilinear DAEs (1.6)-(1.8) (cf. (4.5)) and (1.9)-(1.13).

For shorter expressions we drop the arguments in the following section. In order to distinguish between constant and non-constant terms, we will use a dot as an argument for non-constant terms.

Theorem 4.3 Let the assumptions of Theorem 2.1 be satisfied. Then it holds:

1. If the network contains neither $L-I$ cutsets nor $C$-V loops, then the conventional MNA leads to an index-1 tractable DAE system.

2. If the network contains $L-I$ cutsets or $C$-V loops, then the conventional $M N A$ leads to an index-2 tractable DAE system. The canonical projector $Q_{1}(\cdot)$ onto $N_{1}(x, t)$ along $S_{1}$ is given by

$$
\left(\begin{array}{ccc}
H_{1}^{-1}(\cdot) A_{V} \bar{Q}_{V-C} H_{6}^{-1}(\cdot) \bar{Q}_{V-C}^{T} A_{V}^{T} P_{C} & -P_{C} Q_{V} X(\cdot) Q_{C R V} H_{5}^{-1}(\cdot) Q_{C R V}^{T} A_{L} & 0 \\
0 & L^{-1}(\cdot) A_{L}^{T} Q_{C R V} H_{5}^{-1}(\cdot) Q_{C R V}^{T} A_{L} & 0 \\
& -A_{V}^{T} Y(\cdot) Q_{C R V} H_{5}^{-1}(\cdot) Q_{C R V}^{T} A_{L} & 0
\end{array}\right)
$$

where the matrices $X(\cdot)$ and $Y(\cdot)$ are chosen in such a way that

$$
A_{I b, c} \frac{d i_{b, c}(\cdot)}{d e} Q_{C}=A_{C} C(\cdot) A_{C}^{T} Q_{V} X(\cdot)+A_{V} A_{V}^{T} Y(\cdot) .
$$

Note, the matrices $H_{1}(\cdot), H_{5}(\cdot)$, and $H_{6}(\cdot)$ were defined in Lemma 1.5.

Remark: The existence of such matrices $X(\cdot)$ and $Y(\cdot)$ is satisfied since the relation $Q_{V-C}^{T} Q_{C}^{T} A_{I b, c} \frac{d i_{b, c}(\cdot)}{d e}=0$ is true (cf. (2.5) and (2.7)).

Before we will prove this theorem, we want to consider the special structure of $A(\cdot), B(\cdot), Q(\cdot), S(\cdot), A_{1}(\cdot), B_{1}(\cdot), Q_{1}(\cdot)$, and $S_{1}(\cdot)$ in case of circuit simulation. Writing the system (1.6)-(1.8) as a nonlinear DAE (4.1) with $A\left(x^{\prime}, x, t\right):=$ $f_{x^{\prime}}^{\prime}\left(x^{\prime}, x, t\right)$ and $B\left(x^{\prime}, x, t\right):=f_{x}^{\prime}\left(x^{\prime}, x, t\right)$, we obtain that

$$
A(\cdot)=\left(\begin{array}{ccc}
A_{C} C(\cdot) A_{C}^{T} & 0 & 0 \\
0 & L(\cdot) & 0 \\
0 & 0 & 0
\end{array}\right)
$$


and

$$
B(\cdot)=\left(\begin{array}{ccc}
A_{C} \bar{C}(\cdot) A_{C}^{T}+A_{R} G(\cdot) A_{R}^{T}+A_{I} \frac{d i(\cdot)}{d e} & A_{L}+A_{I} \frac{d i(\cdot)}{d j_{L}} & A_{V}+A_{I} \frac{d i(\cdot)}{d j} \\
-A_{L}^{T} & \bar{L}(\cdot) & 0 \\
A_{V}^{T}-\frac{d v(\cdot)}{d e} & -\frac{d v(\cdot)}{d j_{L}} & 0
\end{array}\right)
$$

with

$$
\bar{C}(\dot{u}, u, t)=\frac{d}{d u} C(u, t) \dot{u}+\frac{d}{d u} q_{t}^{\prime}(u, t)
$$

and

$$
\bar{L}\left(\dot{j}_{L}, j_{L}, t\right)=\frac{d}{d j_{L}} L\left(j_{L}, t\right) \dot{j}_{L}+\frac{d}{d j_{L}} \phi_{t}^{\prime}\left(j_{L}, t\right)
$$

Let us remark here that $A(\cdot)$ represents the leading coefficient matrix. It has a different meaning than the incidence matrix $A=\left(A_{C}, A_{L}, A_{R}, A_{V}, A_{I}\right)$. We will not use the notation $A$ any longer. Therefore, this denotation should be acceptable.

Since $C(\cdot)$ is positive definite, we may conclude that

$$
\operatorname{ker} A(\cdot)=\operatorname{ker} A_{C}^{T} \times\{0\} \times \mathbb{R}^{n_{V}}
$$

and

$$
\operatorname{im} A(\cdot)=\operatorname{im} A_{C} \times \mathbb{R}^{n_{L}} \times\{0\} .
$$

Here, $n_{V}$ describes the number of voltage sources and $n_{L}$ describes the number of inductances in the network. Note, the null-space of $A(\cdot)$ as well as the imagespace of $A(\cdot)$ are constant in any case. The null-space of $A(\cdot)$ describes the nondynamic components of the circuit. For further considerations let us introduce a projector $Q$ onto $\operatorname{ker} A(\cdot)$ as

$$
Q=\left(\begin{array}{ccc}
Q_{C} & 0 & 0 \\
0 & 0 & 0 \\
0 & 0 & I
\end{array}\right)
$$

For the definition of $Q_{C}$ see page 6. The space $S(\cdot)=\{z: B(\cdot) z \in \operatorname{im} A(\cdot)\}$ describes all solution components for which we do not find an algebraic representation. Regarding (4.6) it is given by

$$
\begin{aligned}
& S(\cdot)=\left\{z:\left(A_{V}^{T}-\frac{d v(\cdot)}{d e}\right) z_{e}-\frac{d v(\cdot)}{d j_{L}} z_{L}=0\right. \\
& \left.\left(A_{R} G(\cdot) A_{R}^{T}+A_{I} \frac{d i(\cdot)}{d e}\right) z_{e}+\left(A_{L}+A_{I} \frac{d i(\cdot)}{d j_{L}}\right) z_{L}+\left(A_{V}+A_{I} \frac{d i(\cdot)}{d j_{V}}\right) z_{V} \in \operatorname{im} A_{C}\right\} .
\end{aligned}
$$

Consider the space $N \cap S(\cdot)$. It represents all components that are determined neither by a differential equation nor by an algebraic equation. If $N \cap S(\cdot) \neq$ $\{0\}$, then these components can be determined only by inherent differentiation instead of integration. The next lemma provides a possibility to determine from the network topology whether a differentiation problem is involved in the DAE 4.1 obtained applying MNA. This has a big influence onto numerical solving since differentiation problems are ill-posed in the sense of Hadamard, i.e., small perturbations in the input data can provide arbitrarily large perturbations in the output data. 
Lemma 4.4 Let the conditions of Theorem 2.1 be satisfied. Then it holds that

$$
N \cap S(\cdot)=\operatorname{im}\left(\begin{array}{ccc}
Q_{C R V} & 0 & 0 \\
0 & 0 & 0 \\
-\bar{P}_{V-C} Z(\cdot) Q_{C R V} & 0 & \bar{Q}_{V-C}
\end{array}\right)
$$

is true for a matrix $Z(\cdot)$ satisfying $Q_{C}^{T} A_{I c} \frac{d i_{c}(\cdot)}{d e}=Q_{C}^{T} A_{V} Z(\cdot)$.

\section{Remarks:}

1. The existence of such a matrix $Z(\cdot)$ is guaranteed by condition $(2 \mathrm{c})$ of Theorem 2.1 (cf. (2.7)).

2. Regarding the definitions of $Q_{C R V}$ and $\bar{Q}_{V-C}$ on page 6 as well as Theorem 1.1, Lemma 4.4 implies that the network equations involve a differentiation problem if and only if the network contains a C-V loop or an L-I cutset.

3. If all controlled current sources satisfy the conditions (2a) or (2b) of Theorem 2.1, then the relation

$$
N \cap S=\operatorname{im} Q_{C R V} \times\{0\} \times \operatorname{im} \bar{Q}_{V-C}
$$

is true.

4. The different structure of the general case and the one discussed in the latter point corresponds to the alteration of the order in which we solve the system for the differential index (cf. footnote 5). At this point it is recognizable that $N \cap S(\cdot)$ represents those components for which the differential index definition requires two differentiations to obtain the representation of their derivative as a continuous function of the variables.

Proof: Firstly, we show that the relation "ᄃ" is true. Assuming $z \in N \cap S(\cdot)$ we know that $z_{e}=Q_{C} z_{e}, z_{L}=0$ and $z \in \bar{S}(\cdot)$. Using (2.2), (2.4), (2.5), and (2.8) we obtain

$$
\begin{aligned}
A_{V}^{T} z_{e} & =0 \\
Q_{C}^{T} A_{R} G(\cdot) A_{R}^{T} Q_{C} z_{e}+Q_{C}^{T} A_{I c} \frac{d i_{c}(\cdot)}{d e} z_{e}+Q_{C}^{T} A_{V} z_{V} & =0
\end{aligned}
$$

Then, equation (4.7) provides additionally that $Q_{V-C}^{T} Q_{C}^{T} A_{I c} \frac{d i_{c}(\cdot)}{d e} z_{e}=0$ (cf. (2.7)). Multiplying (4.8) by $Q_{V-C}^{T}$ and regarding $A_{V}^{T} Q_{C} z_{e}=0$ we obtain

$$
Q_{V-C}^{T} Q_{C}^{T} A_{R} G(\cdot) A_{R}^{T} Q_{C} Q_{V-C} z_{e}=0 .
$$

Since $G(\cdot)$ is positive definite, this implies $A_{R}^{T} Q_{C} Q_{V-C} z_{e}=0$, i.e., $A_{R}^{T} z_{e}=0$ and so $z_{e} \in \operatorname{im} Q_{C R V}$. Now the relation (4.8) implies that

$$
\begin{aligned}
& Q_{C}^{T} A_{V} z_{V}=-Q_{C}^{T} A_{I c} \frac{d i_{c}(\cdot)}{d e}=-Q_{C}^{T} A_{V} Z(\cdot) z_{e}=-Q_{C}^{T} A_{V} Z(\cdot) Q_{C R V} z_{e} \\
& \text { i.e., } \\
& \qquad z_{V}=-\bar{P}_{V-C} Z(\cdot) Q_{C R V} z_{e}+\bar{Q}_{V-C} z_{V} .
\end{aligned}
$$


Secondly, we show that the relation " $\supseteq$ " is satisfied. Assume that $z_{e}=Q_{C R V} z_{e}$ and $z_{L}=0$. Furthermore, we have

$$
z_{V}=\bar{Q}_{V-C} z_{V}-\bar{P}_{V-C} Z(\cdot) z_{e} .
$$

Then $z \in N=\operatorname{ker} A(\cdot)$ holds trivially and

$$
\left(A_{V}^{T}-\frac{d v(\cdot)}{d e}\right) z_{e}-\frac{d v(\cdot)}{d j_{L}} z_{L}=\frac{d v(\cdot)}{d e} Q_{C R V} z_{e}=0
$$

is fulfilled. Using (4.9) we obtain additionally that

$$
\begin{array}{r}
Q_{C}^{T}\left[\left(A_{R} G(\cdot) A_{R}^{T}+A_{I} \frac{d i(\cdot)}{d e}\right) z_{e}+\left(A_{L}+A_{I} \frac{d i(\cdot)}{d j_{L}}\right) z_{L}+\left(A_{V}+A_{I} \frac{d i(\cdot)}{d j_{V}}\right) z_{V}\right] \\
=Q_{C}^{T}\left[A_{I c} \frac{d i_{c}(\cdot)}{d e} z_{e}+A_{V} z_{V}\right]=Q_{C}^{T} A_{V} Z(\cdot) z_{e}+Q_{C}^{T} A_{V} z_{V}=0
\end{array}
$$

q.e.d.

Corollary 4.5 Let the conditions of Theorem 2.1 be satisfied. The network equation system obtained applying MNA is index-1-tractable if and only if the network contains neither a $C$-V loop nor an $L-I$ cutset.

Let us now study the higher index case. For that we investigate $A_{1}(\cdot)$ and $B_{1}(\cdot)$ defined on page 19 .

$$
\begin{gathered}
A_{1}(\cdot)=\left(\begin{array}{ccc}
A_{C} C(\cdot) A_{C}^{T}+A_{R} G(\cdot) A_{R}^{T} Q_{C}+A_{I} \frac{d i(\cdot)}{d e} Q_{C} & 0 & A_{V}+A_{I} \frac{d i(\cdot)}{d j_{V}} \\
-A_{L}^{T} Q_{C} & L(\cdot) & 0 \\
A_{V}^{T} Q_{C} & 0 & 0
\end{array}\right) \\
B_{1}(\cdot)=\left(\begin{array}{ccc}
A_{C} \bar{C}(\cdot) A_{C}^{T}+A_{R} G(\cdot) A_{R}^{T} P_{C}+A_{I} \frac{d i(\cdot)}{d e} P_{C} & A_{L}+A_{I} \frac{d i(\cdot)}{d j_{L}} & 0 \\
-A_{L}^{T} P_{C} & \bar{L}(\cdot) & 0 \\
A_{V}^{T} P_{C}-\frac{d v(\cdot)}{d e} P_{C} & 0 & 0
\end{array}\right)
\end{gathered}
$$

Lemma 4.6 Let the conditions of Theorem 2.1 be satisfied. Then the relation

$$
\operatorname{im} A_{1}(\cdot)=\operatorname{ker}\left(\begin{array}{ccc}
Q_{C R V}^{T} & 0 & 0 \\
0 & 0 & 0 \\
0 & 0 & \bar{Q}_{V-C}^{T}
\end{array}\right)
$$

is satisfied.

Proof: Firstly, im $A_{1}(\cdot) \subseteq \operatorname{ker} Q_{C R V}^{T} \times \mathbb{R}^{n_{L}} \times \operatorname{ker} \bar{Q}_{V-C}^{T}$ holds trivially, because of

$$
Q_{C R V}^{T} A_{I} \frac{d i(\cdot)}{d e}=0 \quad \text { and } \quad Q_{C R V}^{T} A_{I} \frac{d i(\cdot)}{d j_{V}}=0
$$

for all admitted controlled current sources (see (2.3), (2.5), and (2.7)). Secondly, we assume that $z \in \operatorname{ker} Q_{C R V}^{T} \times \mathbb{R}^{n_{L}} \times \operatorname{ker} \bar{Q}_{V-C}^{T}$, i.e., $Q_{C R V}^{T} z_{1}=0$ and $\bar{Q}_{V-C}^{T} z_{3}=0$. Then, there is an $\alpha_{0}$ such that

$$
z_{3}=A_{V}^{T} Q_{C} \alpha_{0} \text {. }
$$


Since $Q_{C R V}^{T} A_{I}=0$ (see $(2.3),(2.5)$, and (2.7)), the relation

$$
z_{1}-A_{R} G(\cdot) A_{R}^{T} Q_{C} P_{V-C} \alpha_{0}-A_{I} \frac{d i(\cdot)}{d e} Q_{C} P_{V-C} \alpha_{0} \in \operatorname{ker} Q_{C R V}^{T}
$$

holds, i.e., there are $\alpha_{1}, \alpha_{2}$ and $\gamma_{1}$ such that

$$
\begin{aligned}
& z_{1}-A_{R} G(\cdot) A_{R}^{T} Q_{C} P_{V-C} \alpha_{0}-A_{I} \frac{d i(\cdot)}{d e} Q_{C} P_{V-C} \alpha_{0} \\
& =A_{C} C(\cdot) A_{C}^{T} \alpha_{1}+A_{R} G(\cdot) A_{R}^{T} Q_{C} Q_{V-C} \alpha_{2}+A_{V} \gamma_{1} .
\end{aligned}
$$

This is a simple conclusion of the fact that

$$
\operatorname{ker} Q_{C R V}^{T}=\operatorname{im}\left(A_{C} C(\cdot) A_{C}^{T}, A_{R} G(\cdot) A_{R}^{T} Q_{C} Q_{V-C}, A_{V} A_{V}^{T}\right),
$$

since $C(\cdot)$ and $G(\cdot)$ are positive definite. Regarding (2.4), (2.6), and (2.8) we obtain that

$$
A_{I} \frac{d i(\cdot)}{d j_{V}}=A_{I b} \frac{d i_{b}(\cdot)}{d j_{V}} \bar{P}_{V-C}
$$

Considering (2.3) we see that

$$
\frac{d i_{a}(\cdot)}{d e} Q_{C}=\frac{d i_{a}\left(A_{C}^{T} e, A_{V}^{T} e, j_{L}, t\right)}{d e} Q_{C}=\frac{d i_{a}(\cdot)}{d e} Q_{C} P_{V-C} .
$$

Regarding (2.7) we find $\alpha_{3}$ and $\gamma_{2}$ such that

$$
A_{I c} \frac{d i_{c}(\cdot)}{d e} Q_{C} Q_{V-C} \alpha_{2}=A_{C} C(\cdot) A_{C}^{T} \alpha_{3}+A_{V} \gamma_{2} .
$$

Using (2.5) we find $\alpha_{4}$ and $\alpha_{5}$ such that

$$
\begin{aligned}
A_{I b} \frac{d i_{b}(\cdot)}{d e} Q_{C} Q_{V-C} \alpha_{2} & =A_{C} C(\cdot) A_{C}^{T} \alpha_{4}, \\
A_{I b} \frac{d i_{b}(\cdot)}{d j_{V}}\left(\gamma_{1}-\gamma_{2}\right) & =A_{C} C(\cdot) A_{C}^{T} \alpha_{5} .
\end{aligned}
$$

Choosing $\alpha:=P_{C}\left(\alpha_{1}-\alpha_{3}-\alpha_{4}-\alpha_{5}\right)+Q_{C} P_{V-C} \alpha_{0}+Q_{C} Q_{V-C} \alpha_{2}, \beta:=$ $L^{-1}(\cdot)\left(z_{2}+A_{L}^{T} Q_{C} \alpha\right), \gamma:=\gamma_{1}-\gamma_{2}$ and regarding (4.11)-(4.16), we obtain that

$$
z=A_{1}(\cdot)\left(\begin{array}{l}
\alpha \\
\beta \\
\gamma
\end{array}\right) \in \operatorname{im} A_{1}(\cdot) .
$$

q.e.d.

Considering (4.2) and Lemma 4.6 we obtain a simple description of the constant space $S_{1}$ :

$$
S_{1}=\operatorname{ker} \bar{Q}_{V-C}^{T} A_{V}^{T} P_{C} \times \operatorname{ker} Q_{C R V}^{T} A_{L} \times \mathbb{R}^{n_{V}} .
$$

For a definition of $S_{1}$ see page 19 . 
Lemma 4.7 Let the conditions of Theorem 2.1 be satisfied. Then, the canonical projector onto $N_{1}(\cdot)=\operatorname{ker} A_{1}(\cdot)$ along $S_{1}$ is given by (4.2).

\section{Proof:}

1. $Q_{1}(\cdot)$ is a projector, since

$$
\begin{aligned}
\bar{Q}_{V-C} H_{6}^{-1}(\cdot) & =H_{6}^{-1}(\cdot) \bar{Q}_{V-C}^{T}, \\
H_{6}^{-1}(\cdot) \bar{Q}_{V-C}^{T} A_{V}^{T} H_{1}^{-1}(\cdot) A_{V} \bar{Q}_{V-C} & =\bar{Q}_{V-C}, \\
P_{C} Q_{C R V} & =0 \\
Q_{C R V} H_{5}^{-1}(\cdot) & =H_{5}^{-1}(\cdot) Q_{C R V}^{T}, \text { and } \\
H_{5}^{-1}(\cdot) Q_{C R V}^{T} A_{L} L^{-1}(\cdot) A_{L}^{T} Q_{C R V} & =Q_{C R V} .
\end{aligned}
$$

These relations are simple conclusions of the definitions of $H_{1}(\cdot), H_{5}(\cdot)$, and $H_{6}(\cdot)$.

2. We show that $\operatorname{im} Q_{1}(\cdot) \subseteq \operatorname{ker} A_{1}(\cdot)$. Regarding

$$
\begin{aligned}
A_{C} C(\cdot) A_{C}^{T} H_{1}^{-1}(\cdot) & =P_{C}^{T} \\
Q_{C} H_{1}^{-1}(\cdot) & =H_{1}^{-1}(\cdot) Q_{C}^{T} \\
\frac{d i(\cdot)}{d j_{V}} & =\frac{d i_{b}(\cdot)}{d j_{V}} \bar{P}_{V-C}
\end{aligned}
$$

this holds trivially.

3. We show that $\operatorname{ker} A_{1}(\cdot) \subseteq \operatorname{im} Q_{1}(\cdot)$. Assume $z \in \operatorname{ker} A_{1}(\cdot)$. Then,

$$
\begin{aligned}
A_{C} C(\cdot) A_{C}^{T} z_{e}+A_{R} G(\cdot) A_{R}^{T} Q_{C} z_{e}+A_{I} \frac{d i(\cdot)}{d e} Q_{C} z_{e} & \\
+A_{V} z_{V}+A_{I} \frac{d i(\cdot)}{d j} z_{V} & =0 \\
-A_{L}^{T} Q_{C} z_{e}+L(\cdot) z_{L} & =0 \\
A_{V}^{T} Q_{C} z_{e} & =0 .
\end{aligned}
$$

Considering (4.21) we see that

$$
z_{e}=Q_{V-C} z_{e}
$$

Next we have (cf. (2.4), (2.5), and (2.7))

$$
\begin{aligned}
A_{I a} \frac{d i_{a}(\cdot)}{d e} Q_{C} z_{e} & =0, & A_{I a} \frac{d i_{a}(\cdot)}{d j_{V}} z_{V} & =0, \\
Q_{C}^{T} A_{I b} \frac{d i_{b}(\cdot)}{d e} Q_{C} z_{e} & =0, & Q_{C}^{T} A_{I b} \frac{d i_{b}(\cdot)}{d j_{V}} z_{V} & =0, \\
Q_{V-C}^{T} Q_{C}^{T} A_{I c} \frac{d i_{c}(\cdot)}{d e} Q_{C} z_{e} & =0, & Q_{V-C}^{T} Q_{C}^{T} A_{I c} \frac{d i_{c}(\cdot)}{d j_{V}} z_{V} & =0 .
\end{aligned}
$$

Multiplying (4.19) by $Q_{V-C}^{T} Q_{C}^{T}$ yields

$$
Q_{V-C}^{T} Q_{C}^{T} A_{R} G(\cdot) A_{R}^{T} Q_{C} Q_{V-C} z_{e}=0 .
$$


Since $G(\cdot)$ is positive definite and $(4.22)$ is valid, it holds that

$$
Q_{C} z_{e}=Q_{C R V} z_{e}
$$

Relation (4.20) leads to

$$
z_{L}=L^{-1}(\cdot) A_{L}^{T} Q_{C} z_{e}=L^{-1}(\cdot) A_{L}^{T} Q_{C R V} z_{e} .
$$

Multiplying (4.19) by $Q_{C}^{T}$ yields now

$$
Q_{C}^{T} A_{V} z_{V}+Q_{C}^{T} A_{I c} \frac{d i_{c}(\cdot)}{d e} Q_{C} z_{e}=0 .
$$

If we regard (4.23)-(4.25), then the relation 4.19) reduces to

$$
A_{C} C(\cdot) A_{C}^{T} z_{e}+A_{I b, c} \frac{d i_{b, c}(\cdot)}{d e} Q_{C} z_{e}+A_{V} z_{V}=0 .
$$

Since $A_{I b, c} \frac{d i_{b, c}(\cdot)}{d e} Q_{C}=A_{C} C(\cdot) A_{C}^{T} Q_{V} X(\cdot)+A_{V} A_{V}^{T} Y(\cdot)$, we obtain that

$$
Q_{C}^{T} A_{I b, c} \frac{d i_{b, c}(\cdot)}{d e}=Q_{C}^{T} A_{V} A_{V}^{T} Y(\cdot) .
$$

Multiplying (4.28) by $Q_{C}^{T}$ we conclude that

$$
Q_{C}^{T} A_{V}\left(A_{V}^{T} Y(\cdot) Q_{C} z_{e}+z_{V}\right)=0
$$

i.e.,

$$
A_{V}^{T} Y(\cdot) Q_{C} z_{e}+z_{V}=\bar{Q}_{V-C}\left(A_{V}^{T} Y(\cdot) Q_{C} z_{e}+z_{V}\right) .
$$

From (4.28) we obtain

$$
\begin{aligned}
A_{C} C(\cdot) A_{C}^{T} z_{e}+A_{C} C(\cdot) A_{C}^{T} Q_{V} X(\cdot) Q_{C} z_{e} & \\
+A_{V} A_{V}^{T} Y(\cdot) Q_{C} z_{e}+A_{V} z_{V} & =0
\end{aligned}
$$

i.e.,

$$
\begin{aligned}
P_{C}\left(z_{e}+Q_{V} X(\cdot) Q_{C} z_{e}\right) & =-H_{1}^{-1}(\cdot) A_{V}\left(z_{V}+A_{V}^{T} Y(\cdot) Q_{C} z_{e}\right) \\
& =-H_{1}^{-1}(\cdot) A_{V} \bar{Q}_{V-C}\left(z_{V}+A_{V}^{T} Y(\cdot) Q_{C} z_{e}\right)
\end{aligned}
$$

because of (4.29). Thus

$$
z=Q_{1}(\cdot)\left(\begin{array}{c}
z_{e}+Q_{V} X(\cdot) Q_{C} z_{e} \\
L^{-1}(\cdot) A_{L}^{T} Q_{C R V} z_{e} \\
0
\end{array}\right) \in \operatorname{im} Q_{1}(\cdot) .
$$

4. The relation $S_{1} \subseteq \operatorname{ker} Q_{1}(\cdot)$ is a simple conclusion of (4.18).

5. We show that $\operatorname{ker} Q_{1}(\cdot) \subseteq S_{1}$. Assume $Q_{1}(\cdot) z=0$. Then

$$
L^{-1} A_{L}^{T} Q_{C R V} H_{5}^{-1}(\cdot) Q_{C R V}^{T} A_{L} z_{L}=0
$$

and

$$
\bar{Q}_{V-C} H_{6}^{-1}(\cdot) \bar{Q}_{V-C}^{T} A_{V}^{T} P_{C} z_{e}=0 .
$$

Multiplying (4.30) by $Q_{C R V}^{T} A_{L}$ yields $Q_{C R V}^{T} A_{L} z_{L}=0$. Regarding

$$
\bar{Q}_{V-C} H_{6}^{-1}(\cdot)=H_{6}^{-1}(\cdot) \bar{Q}_{V-C}^{T}
$$

we conclude from (4.30) that $\bar{Q}_{V-C}^{T} A_{V}^{T} P_{C} z_{e}=0$. Considering (4.18) the assertion is proved. 
q.e.d

Remark: Lemma 4.4 and Lemma 4.7 now imply Theorem 4.3 for the tractability index.

The validity of Theorem 2.2 follows by similar considerations as above. More precisely, it can be shown that the following relations are satisfied for the chargeoriented MNA.

(i) The analogue to Lemma 4.4 reads

$$
N \cap S(\cdot)=\operatorname{im}\left(\begin{array}{ccccc}
0 & 0 & 0 & 0 & 0 \\
0 & 0 & 0 & 0 & 0 \\
0 & 0 & Q_{C R V} & 0 & 0 \\
0 & 0 & 0 & 0 & 0 \\
0 & 0 & -\bar{P}_{V-C} Z(\cdot) Q_{C R V} & 0 & \bar{Q}_{V-C}
\end{array}\right)
$$

for $Z(\cdot)$ chosen as in Lemma 4.4.

(ii) Lemma 4.6 reads

$$
\operatorname{im} A_{1}(\cdot)=\operatorname{ker}\left(\begin{array}{ccccc}
Q_{C R V}^{T} & 0 & 0 & 0 & Q_{C R V}^{T} A_{L} L^{-1}(\cdot) \\
0 & 0 & 0 & 0 & 0 \\
0 & 0 & \bar{Q}_{V-C}^{T} & \bar{Q}_{V-C}^{T} A_{V}^{T} H_{1}^{-1}(\cdot) A_{C} & 0 \\
0 & 0 & 0 & 0 & 0 \\
0 & 0 & 0 & 0 & 0
\end{array}\right)
$$

(iii) Equation (4.18) corresponds to

$$
S_{1}(\cdot)=\operatorname{ker} \bar{Q}_{V-C}^{T} A_{V}^{T} H_{1}^{-1}(\cdot) A_{C} \times \operatorname{ker} Q_{C R V}^{T} A_{L} L^{-1}(\cdot) \times \mathbb{R}^{n_{e}} \times \mathbb{R}^{n_{L}} \times \mathbb{R}^{n_{V}} .
$$

(iv) Theorem 4.3 holds analogously for the charge-oriented MNA, and the canonical projector $Q_{1}(\cdot)$ onto $N_{1}(\cdot)$ along $S_{1}(\cdot)$ is given by

$$
\left(\begin{array}{ccccc}
\bar{P}_{C} A_{C}^{T} \bar{H}_{1}^{-1} A_{V} \bar{Q}_{V-C} \cdot(\cdot) & -\bar{P}_{C} C(\cdot) A_{C}^{T} Q_{V} X(\cdot) Q_{C R V} \cdot & 0 & 0 & 0 \\
H_{6}^{-1}(\cdot) \bar{Q}_{V-C}^{T} A_{V}^{T} H_{1}^{-1}(\cdot) A_{C} & H_{5}^{-1}(\cdot) Q_{C R V}^{T} A_{L} L^{-1}(\cdot) & & \\
0 & A_{L}^{T} Q_{C R V} H_{5}^{-1}(\cdot) Q_{C R V}^{T} A_{L} L^{-1}(\cdot) & 0 & 0 & 0 \\
0 & Q_{C R V} H_{5}^{-1}(\cdot) Q_{C R V}^{T} A_{L} L^{-1}(\cdot) & 0 & 0 & 0 \\
0 & 0 & 0 & 0 & 0 \\
-\bar{Q}_{V-C} H_{6}^{-1}(\cdot) \bar{Q}_{V-C}^{T} A_{V}^{T} H_{1}^{-1}(\cdot) A_{C} & -A_{V}^{T} Y(\cdot) Q_{C R V} H_{5}^{-1}(\cdot) Q_{C R V}^{T} A_{L} L^{-1}(\cdot) & 0 & 0 & 0
\end{array}\right)
$$

where $\bar{H}_{1}:=A_{C} A_{C}^{T}+Q_{C}^{T} Q_{C}$ and the matrices $X(\cdot)$ and $Y(\cdot)$ are chosen as before.

Note that if no controlled current sources that fulfill only the conditions (2b) or (2c) of Theorem 2.1 appear, then $N_{1}$ is constant.

\section{Conclusion}

The presented results provide the possibility to obtain information about the index of the systems (1.6)-(1.8) and (1.9)-(1.13) by topological analysis of the 
network. The only assumption made on the parameters defining its elements is the exposed positive definiteness.

The class of controlled sources described in this paper is precisely the one that does not seriously affect the structure of the spaces associated with the DAEsystems. Basically ${ }^{6}$, these spaces are the same as for networks without controlled sources. If no assumptions on the controlled sources are made, then different problems arise.

On the one hand, if arbitrary controlling elements for the controlling sources are considered, then the index of the network equations may depend on the parameters defining them (cf. [13]).

On the other hand, if controlled sources are allowed to form part of L-I-cutsets of $\mathrm{C}-\mathrm{V}$-loops, then it is possible to be confronted with higher index $(>2)$ problems (cf. [9]).

\section{Acknowledgment}

We would like to thank U. Feldmann of the Siemens AG Munich for the encouragement given. We are also indebted to R. März and R. Lamour for the helpful discussions.

\section{References}

[1] K. E. Brenan, S. L. Campbell, and L. R. Petzold. The Numerical Solution of Initial Value Problems in Ordinary Differential-Algebraic Equations. North Holland Publishing Co., 1989.

[2] L. O. Chua and Pen-Min Lin. Computer-Aided Analysis of Electronic Circuits. Prentice Hall, Englewood Cliffs, 1975.

[3] C.A. Desoer and E.S. Kuh. Basic Circuit Theory. McGraw-Hill, Singapore, 1969.

[4] D. Estévez Schwarz. Consistent initialization of differential-algebraic equations in circuit simulation, 1998. In preparation.

[5] M. Fosséprez. Non-linear Circuits: Qualitative Analysis of Non-linear, Non-reciprocal Circuits. John Wiley \& Sons, Chichester, 1992.

[6] E. Griepentrog and R. März. Differential-Algebraic Equations and Their Numerical Treatment. Teubner-Texte zur Mathematik No. 88. BSB B.G. Teubner Verlagsgesellschaft, Leipzig, 1986.

[7] M. Günther and U. Feldmann. The dae-index in electric circuit simulation. Mathematics and Computers in Simulation, 39:573-582, 1995.

[8] M. Günther and U. Feldmann. CAD based electric modeling in industry. Part I : Mathematical structure and index of network equations. To appear in Surv. Math. Ind., 1998.

[9] M. Günther and U. Feldmann. CAD based electric modeling in industry. Part II: Impact of circuit configurations and parameters. To appear in Surv. Math. Ind., 1998.

\footnotetext{
${ }^{6}$ One exception is the one discussed in Lemma 4.4 and footnote 5. Straight forward computation shows that also $N_{1}(\cdot)$ may change depending on the considered controlled sources.
} 
[10] E. Hairer and G. Wanner. Solving Ordinary Differential Equations II: Stiff and differential-algebraic problems. Springer Series in Computational Mathematics 14. Springer-Verlag, Berlin, Heidelberg, 1991.

[11] R. März. Numerical methods for differential-algebraic equations. Acta Numerica, pages 141-198, 1992.

[12] R. März and C. Tischendorf. Recent results in solving index 2 differential algebraic equations in circuit simulation. SIAM J. Sci. Stat. Comput., 18(1):139-159, 1997.

[13] G. Reißig. Beiträge zu Theorie und Anwendung impliziter Differentialgleichungen. PhD thesis, Techn. Univ. Dresden, 1998.

[14] H. Shichman and D. A. Hodges. Insulated-gate field-effect transistor switching circuits. IEEE J. Solid State Circuits, SC-3:285-289, 1968.

[15] C. Tischendorf. Solution of index-2 differential algebraic equations and its application in circuit simulation. PhD thesis, Humboldt-Univ. Berlin, 1996.

[16] C. Tischendorf. Topological index calculation of DAEs in circuit simulation. to appear in Surv. Math. Ind., 1998. 


\section{Notations}

MNA Modified Nodal Analysis,

VCVS voltage-controlled voltage sources,

CCVS current-controlled voltage sources,

VCCS voltage-controlled current sources,

CCCS current-controlled current sources,

L-I cutset cutset consisting of inductances and/or current sources only,

C-V loop loop consisting of capacitances and voltage sources only,

$A=\left(A_{C}, A_{L}, A_{R}, A_{V}, A_{I}\right)$ (reduced) incidence matrix describing the branche-node relations:

$A_{C} \quad$ capacitive branches,

$A_{L}$ inductive branches,

$A_{R}$ resistive branches,

$A_{V}$ branches of voltage sources,

$A_{I} \quad$ branches of current sources,

$Q_{C} \quad$ projector onto $\operatorname{ker} A_{C}^{T}$,

$Q_{V-C} \quad$ projector onto $\operatorname{ker} A_{V}^{T} Q_{C}$,

$Q_{R-C V} \quad$ projector onto $\operatorname{ker} A_{R}^{T} Q_{C} Q_{V-C}$,

$Q_{V} \quad$ projector onto $\operatorname{ker} A_{V}^{T}$,

$\bar{Q}_{C} \quad$ projector onto ker $A_{C}$,

$\bar{Q}_{V-C} \quad$ projector onto $\operatorname{ker} Q_{C}^{T} A_{V}$,

$Q_{C R V}:=Q_{C} Q_{V-C} Q_{R-C V}$,

$C(u, t):=\frac{\partial q(u, t)}{\partial u}, \quad q_{t}^{\prime}(u, t):=\frac{\partial q(u, t)}{\partial t}$,

$L(j, t):=\frac{\partial \phi(j, t)}{\partial j}, \quad \phi_{t}^{\prime}(j, t):=\frac{\partial \phi(j, t)}{\partial t}$,

$G(u, t):=\frac{\partial r(u, t)}{\partial u}, \quad r_{t}^{\prime}(u, t):=\frac{\partial r(u, t)}{\partial t}$,

$H_{1}\left(A_{C}^{T} e, t\right):=A_{C} C\left(A_{C}^{T} e, t\right) A_{C}^{T}+Q_{C}^{T} Q_{C}$,

$H_{2}:=Q_{C}^{T} A_{V} A_{V}^{T} Q_{C}+Q_{V-C}^{T} Q_{V-C}$,

$H_{3}:=A_{V}^{T} Q_{C} Q_{C}^{T} A_{V}+\bar{Q}_{V-C}^{T} \bar{Q}_{V-C}$,

$H_{4}\left(A_{R}^{T} e\right):=Q_{V-C}^{T} Q_{C}^{T} A_{R} G\left(A_{R}^{T} e, t\right) A_{R}^{T} Q_{C} Q_{V-C}+Q_{R-C V}^{T} Q_{R-C V}$,

$H_{5}\left(j_{L}, t\right):=Q_{C R V}^{T} A_{L} L^{-1}\left(j_{L}, t\right) A_{L}^{T} Q_{C R V}+P_{C R V}^{T} P_{C R V}$,

$H_{6}\left(A_{C}^{T} e, t\right):=\bar{Q}_{V-C}^{T} A_{V}^{T} H_{1}^{-1}\left(A_{C}^{T} e, t\right) A_{V} \bar{Q}_{V-C}+\bar{P}_{V-C}^{T} \bar{P}_{V-C}$,

$\bar{H}_{C 1}:=A_{C}^{T} A_{C}+\bar{Q}_{C}^{T} \bar{Q}_{C}$,

$\bar{H}_{C 2}:=\bar{H}_{1}:=A_{C} A_{C}^{T}+Q_{C}^{T} Q_{C}$. 